\title{
Comparative analysis of avascular areas in superficial esophageal squamous cell carcinomas using in vivo and ex vivo magnifying endoscopy
}

\section{다 (우우}

\author{
Authors \\ Institutions \\ 1 Tokyo Medical and Dental University Hospital - \\ Gastrointestinal Surgery, Tokyo, Japan \\ 2 Tokyo Medical and Dental University Hospital, Division \\ of Pathology, Tokyo, Japan
}

Taichi Ogo ${ }^{1}$, Kenro Kawada', Yasuaki Nakajima', Yutaka Tokairin'1, Takashi Ito², Tatsuyuki Kawano ${ }^{1}$

submitted 6.2.2017

accepted after revision 30.6.2017



Corresponding author

Taichi Ogo, Tokyo Ika Shika Daigaku - gastrointestinal surgery, 1-5-45 Bunkyo-ku, Tokyo 113-8519, Japan

Fax: 03-5803-0110

ogotaichi1105@yahoo.co.jp

\section{ABSTRACT}

Background and study aims An avascular area (AVA), one of the microvasculature changes in superficial esophageal cancers, appears when a tumor demonstrates a bulky growth pattern. We aimed to compare endoscopic and histopathological findings by observing formalin-fixed AVA specimens using magnifying endoscopy.

Patients and methods A prospective analysis was conducted on 16 patients with superficial esophageal cancer, including AVA, who underwent endoscopic submucosal dissection (ESD). Magnifying endoscopy and blue laser imaging were used to identify AVAs. After the ESD, the AVA width was measured on formalin-fixed specimens using magnifying endoscopy, and AVA thickness and depth were determined after hematoxylin and eosin staining using microscopy.

Results Mean AVA widths of M1, M2, and M3/SM-lesions were $0.434,0.578$, and $0.835 \mathrm{~mm}$, respectively (M1 vs. $M 2, P=0.16$; $M 2$ vs. M3/SM-, $P=0.07)$. Mean AVA thicknesses of $M 1, M 2$, and M3/SM-lesions were significantly different $(0.176,0.518$, and $0.800 \mathrm{~mm}$; $\mathrm{M} 1$ vs. $\mathrm{M} 2, \mathrm{P}<0.01$; $\mathrm{M} 2$ vs. M3/SM-, $P<0.05)$. There was a significant correlation between AVA width and thickness.

Conclusions AVA size can be measured accurately on formalin-fixed specimens with magnifying endoscopy. AVA thickness can be useful for determining tumor depth.

\section{Introduction}

The esophagus is the only organ where morphological changes in the superficial microvasculature, from normal squamous epithelium to invasive cancer, can be observed in vivo using magnifying endoscopy [1-4]. After a report describing the intra-papillary capillary loop (IPCL), the superficial fine vascular network of intact esophageal mucosa, was published by Inoue et al. [1], many studies have described in detail the microvascular architecture of esophageal superficial carcinoma [2, $3,5,6$ ]. Kumagai et al. [3] measured the caliber of superficial blood vessels injected with Microfil. Using surgically resected specimens, they found a linear relationship between caliber size and depth of invasion. Inoue et al. [1] and Arima et al. [7] described a classification system based on the IPCL, and these classifications are widely used by Japanese endoscopists [8].

Presence of a hypovascular or avascular area (AVA) is one of the criteria for determining the depth of invasion in esophageal squamous cell carcinoma listed in the Japan Esophageal Society's classification based on magnifying endoscopy [9]. Arima et al. [7] reported that the presence of an AVA reflects tumor depth, and occurs when the cancer tissue demonstrates a bulky growth pattern. They reported that the size of the AVA is closely related to the depth of tumor invasion.

However, accurate measurement of AVA size in vivo is difficult, and thus, its estimation remains limited. Furthermore, accurate comparison of histopathological findings with the 

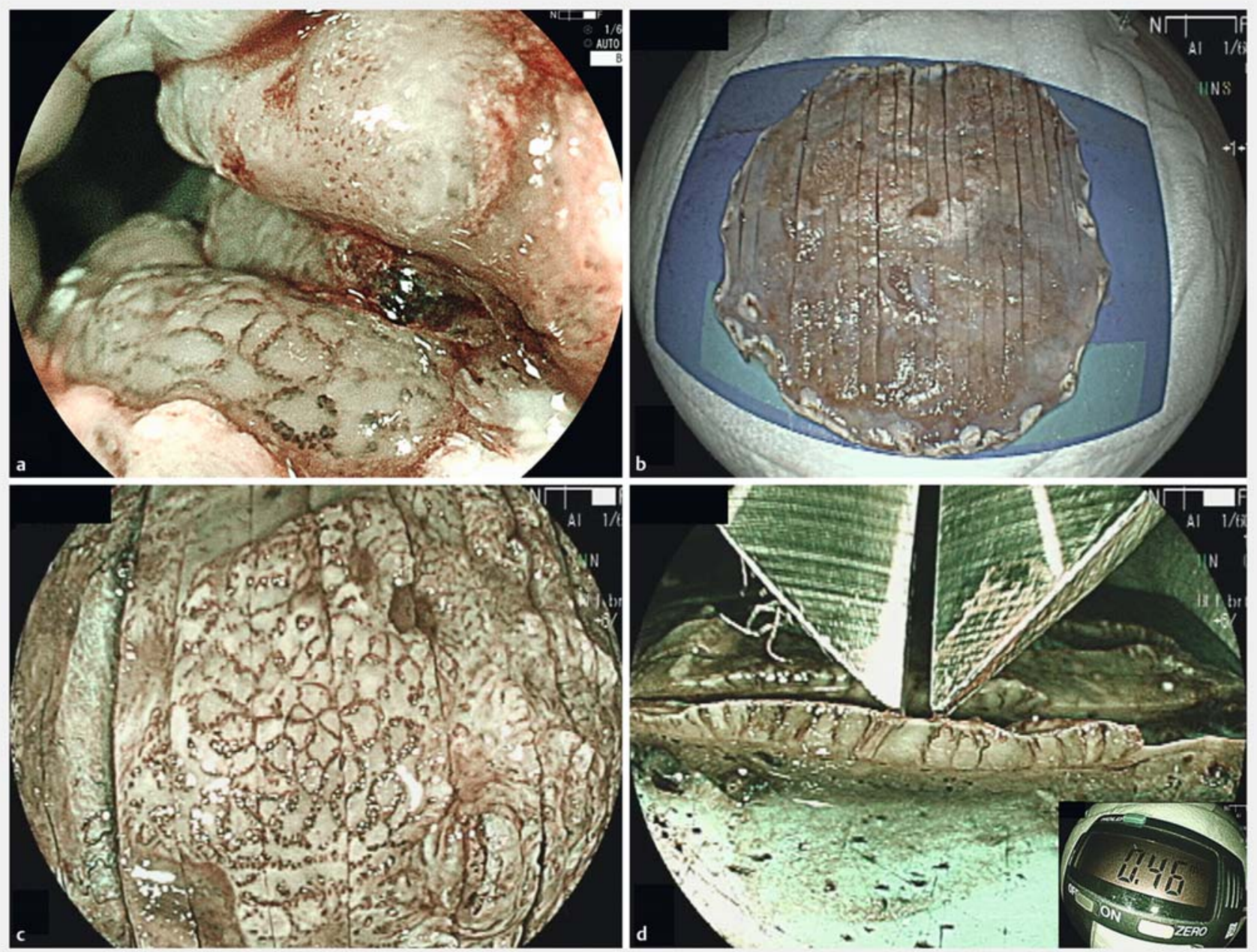

- Fig. 1 Observation and measurement of avascular areas (AVAs). a Magnifying endoscopic image with blue laser imaging under a low-power field. b Formalin-fixed specimens, each with a 2-mm width. c Identification of the concerned areas that had AVAs under a low-power field. d Measurement of the width of the AVAs using fine electronic Vernier calipers.

area of interest observed with magnifying endoscopy is difficult. The current study was undertaken to address these issues by observing formalin-fixed specimens obtained from endoscopic submucosal dissection (ESD) using magnifying endoscopy and determining if accurate evaluation of the microvascular patterns of superficial esophageal cancer is possible with this method.

\section{Patients and methods}

\section{Patient selection}

From October 2013 to September 2015, patients with esophageal squamous cell carcinoma with an AVA detected via magnifying endoscopy, seen at the Esophageal Surgery Department, Tokyo Medical and Dental University Hospital, and who underwent ESD were included in this study. Those who received chemotherapy and/or radiotherapy were excluded from this analysis. For the purpose of this study, AVA was defined as an avascular or hypovascular area surrounded by irregular micro- vessels. The width of the AVA was calculated as the distance of the widest interval between the irregular microvessels.

During the study period, we performed magnifying endoscopy in 122 cases, wherein we detected 139 superficial esophageal squamous cell carcinoma lesions. Among these cases, $23(16.5 \%)$ AVA lesions were detected. Three cases of AVA were not included in this study owing to inadequate preoperative examinations.

Twenty patients with AVA lesions (type 0 -Ilc, $n=14$; type 0 -Ilc +0 -IIa, $n=5$; type 0 -IIc +0 -Is, $n=1$ ) were included in this study. The study population comprised 17 men and 3 women with a mean age of $65.6 \pm 8.2$ (standard deviation [SD]) years (range, 41 - 78 years).

All 20 patients provided informed consent for the ESD and for the use of their resected tumor samples for research purposes through a notice board placed in our hospital's outpatient area. The study was performed under a protocol approved by our hospital ethics committee (registration number: M2015555). 


\section{Materials and procedure}

Magnifying endoscopy with blue laser imaging (BLI) (EGL590ZW; Fujifilm, Tokyo, Japan) was used for endoscopic diagnosis and identification of the AVAs ( $\triangleright$ Fig. 1 a). A soft black hood was attached to the tip of the scope to obtain the appropriate distance from the lesions and to focus on the surface of the lesions accurately during magnification.

ESD was performed in the Department of Endoscopic Diagnostics and Therapeutics under sedation or in the operating room under general anesthesia. After iodine staining and marking the borders of the lesion, glycerol solution ( $10 \%$ glycerol $300 \mathrm{~mL}$ ) along with indigo carmine $(0.6 \mathrm{~mL})$ and $0.1 \%$ adrenaline $(0.6 \mathrm{~mL})$ was injected into the submucosal layer to lift the lesion from the muscularis propria. The incision of the mucosa started at the distal margin of the lesion followed by proximal extension with a flush knife (Fujifilm, Tokyo, Japan). Then, submucosal dissection was performed using the flush knife and Mucosectom (Pentax, Tokyo, Japan). The resected lesion was extended, stuck on a board, and fixed with formalin.

\section{Magnifying observation after formalin fixation}

Two to 3 days after the specimens were resected and fixed with formalin, they were sliced, each with a 2-mm width, without cutting through the specimen completely, at the Division of Pathology ( $\triangleright$ Fig. 1 b). Then, the microvascular structure of the lesions was observed with magnifying endoscopy. The surface of the lesions was examined and AVAs were determined under a low-power field ( $\triangleright$ Fig. $\mathbf{1 ~ c}$ ); then the area was observed under a high-power field.

If the cut line passed through the AVA, a cross-sectional view of the area was obtained via magnifying endoscopy with BLI. The AVA width with the widest interval across the slice was measured using fine electronic Vernier calipers ( $>$ Fig.1 d). To avoid formalin contamination of the sample, we made sure to wipe off the formalin from the specimens before observation, conduct the observation in a short period under well-ventilated conditions, and clean the endoscope immediately after observation in compliance with the in-hospital rule.

\section{Histopathological analysis}

Histopathological specimens were stained with hematoxylin and eosin to assess the depth of tumor invasion. We informed the pathologists which specimen slides had AVAs, who then confirmed the depth of the concerned area. The thickness of the AVAs that had the widest interval was measured using a microscope at $\times 100$ magnification before pathological examination.

Tumor invasion was categorized as M1 (cancer limited to the epithelium), M2 (cancer invading the lamina propria mucosa), M3 (cancer reaching or invading the muscularis mucosa), SM1 (cancer invading submucosa $<200 \mu \mathrm{m}$ ), or SM2 (cancer invading submucosa $>200 \mu \mathrm{m}$ ).
All 23 cases

- Underwent ESD between October 2013 and September 2015

- Superficial esophageal squamous cell carcinoma

- No preoperative therapy

- AVA detected with magnifying endoscopy prior to ESD

3 cases excluded

- Inadequate preoperative examination

20 cases included

4 cases excluded

- Traumatic damage during ESD in 3 cases

- Final diagnosis of basaloid squamous cell carcinoma in 1 case

16 cases analyzed

- Fig. 2 Flowchart of all cases analyzed.

\section{Statistical analysis}

Statistical data are expressed as the mean and $95 \%$ confidence interval. The statistical significance of the differences between groups was analyzed with the Mann-Whitney $U$ test. The correlation between AVA width and thickness was analyzed using Spearman's rank correlation coefficient. $P$ values $<0.05$ were considered statistically significant. Data analysis was performed using the statistical package Ekuseru-Toukei 2012 (Social Survey Research Information Co., Ltd., Tokyo, Japan).

\section{Results}

Eighteen lesions from 16 tissue samples obtained from patients who underwent ESD for superficial esophageal cancer with AVAs were examined and included in this study. Four cases were excluded because in 3 cases, the AVA lesions were lost after formalin fixation owing to traumatic damage during the ESD, and in 1 case, the pathologist's final diagnosis was basaloid-squamous cell carcinoma ( $\boldsymbol{\nabla}$ Fig. 2 ). Eighteen AVA lesions that were clearly visible on BLI after formalin fixation were examined histologically from these groups of patients. The number of lesions in the analyzed area based on depth of invasion was as follows: M1, 5 lesions; M2, 9 lesions; M3, 3 lesions; SM1, 1 lesion.

The tumors' endoscopic and histopathological appearance is shown in > Fig. 3. The M1 lesions formed small AVAs surrounded by crushed spot-like microvessels with irregular calibers. Tumor thickness was very small at this area. The M2 lesions showed expansive downward or upward growth patterns and formed slightly bigger AVAs than the M1 lesions, which did not reach statistical significance. The M3 or SM1 lesions usually had stretched and irregularly branched vessels at the surface of the lesion and appeared thickened ( $>$ Table $\mathbf{1})$. 



- Fig. 3 Endoscopic and histopathological appearance of tumors. a, b, c Magnifying endoscopic images with blue laser imaging (BLI) in vivo. $\mathbf{d}, \mathbf{e}, \mathbf{f}$ Side view of the concerned areas under magnifying endoscopy with BLI after formalin fixation. $\mathbf{g}, \mathbf{h}, \mathbf{i}$ Histopathological features after staining with hematoxylin and eosin (×100 magnification). a, d, $\mathbf{g}$ The M1 lesion was flat and formed a small avascular area. $\mathbf{b}, \mathbf{e}, \mathbf{h}$ The M2 lesion was slightly thickened and showed an expansive growth pattern. c, f, i The SM1 lesion had stretched and irregularly branched vessels at the surface and inside of the lesion. The widest interval between vessels at the surface across the slice was measured.

- Table 1 Comparison of AVA width and thickness among M1, M2, and M3/SM1 lesions.

\begin{tabular}{|l|l|l|l|}
\hline & $\mathbf{n}$ & Width $(\mathbf{m m})$ & Thickness $(\mathbf{m m})$ \\
\hline M1 & 5 & $0.434(0.390-0.478)$ & $0.176(0.119-0.233)$ \\
\hline M2 & 9 & $0.578(0.451-0.705)$ & $0.518(0.390-0.646)$ \\
\hline M3/SM1 & 4 & $0.835(0.601-1.069)$ & $0.800(0.652-0.948)$ \\
\hline
\end{tabular}

Values are presented as the mean (95\% confidence interval).

- Fig. 4 a shows the relationship between the depth of tumor invasion and AVA width as measured using Vernier calipers with magnifying endoscopy. The differences among the three groups were not significant. > Fig. $\mathbf{4 b}$ shows the relationship between the depth of tumor invasion and AVA thickness measured on the microscope at $\times 100$ magnification. The differences between $\mathrm{M} 1$ and $\mathrm{M} 2$ and between $\mathrm{M} 2$ and $\mathrm{M} 3 / \mathrm{SM} 1$ lesions were significant. > Fig. 5 shows the significant relationship between AVA width and thickness.

\section{Discussion}

Recently, several superficial esophageal cancers have been detected owing to improvements in digestive endoscopy. Specifically, image-enhanced endoscopy with magnification including narrow-band imaging, BLI, and flexible spectral imaging color enhancement has enabled clear visualization of the microsvasculature of the tumor surface [5,10-12], which led to the discovery that changes in microvasculature reflect the depth of superficial esophageal cancer [2-4]. 


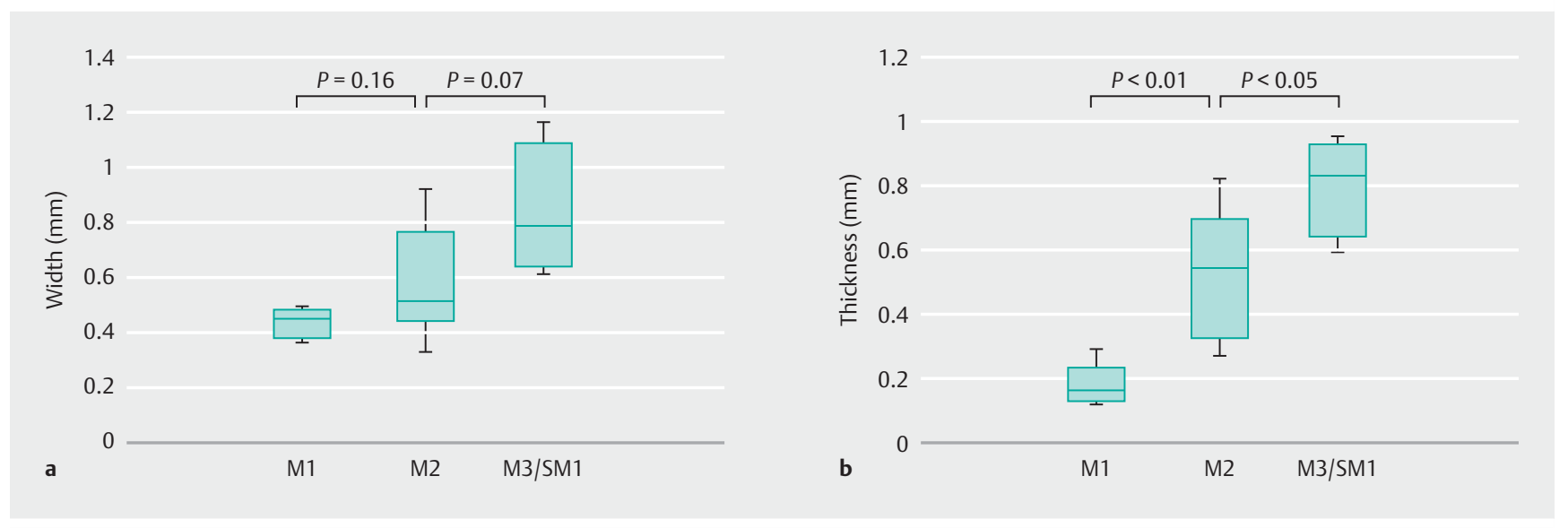

- Fig. 4 a Relationship between avascular area (AVA) width measured using Vernier calipers with magnifying endoscopy and depth of tumor invasion. b Relationship between AVA thickness measured using a microscope and depth of tumor invasion.

A previous study on AVA confirmed that tumor invasion becomes deeper as the size of the AVA increases [7]. In the Japan Esophageal Society classification of magnifying endoscopy findings, AVAs are categorized as AVA-small $(<0.5 \mathrm{~mm}$, corresponds to M1-M2), AVA-middle $(0.5-3.0 \mathrm{~mm}$, corresponds to M3-SM1), and AVA-large ( $>3.0 \mathrm{~mm}$, corresponds to SM2 or deeper). In our study, all $5 \mathrm{M} 1$ lesions were classified as AVAsmall, $4 \mathrm{M} 2$ lesions (total $=9$ lesions) were AVA-small, and the other 5 lesions were AVA-middle. All M3 and SM1 lesions were classified as AVA-middle.

The results of this study corroborate the findings of previous reports comparing the endoscopic observation and estimation of AVA size with histopathological findings by directly measuring the size of the AVAs after formalin fixation. This is the first attempt to evaluate the size of the AVAs using endoscopically resected specimens, which allows for more intensive inspection than does an in vivo study. In addition, our results reveal that the thickness of the AVAs reflect the depth of the tumor. However, the relationship between AVA width and depth of tumor invasion could not be confirmed, presumably owing to the small number of patients. Meanwhile, this report demonstrates the detailed correspondence between AVA width and thickness. Although this study has the limitations of small number of cases, traumatizing the specimen during the procedure, and ex vivo study after formalin fixation, these results are important when we consider AVA formation and development.

AVAs form as the tumor develops expansively and pushes the IPCL sideways. Then, the tumor breaks through the basal membrane and invades a deeper layer. When the tumor reaches a certain size, the center of the tumor becomes ischemic and needs neovascularization [13]. Experimental studies have shown that tumors rarely grow to greater than 2 or $3 \mathrm{~mm}^{3}$ without neovascularization [14]. Thus, there is a limit to the interval between vessels. Consequently, when the tumor's mass reaches a certain size and the tumor develops neovascularization, it can be presumed that the tumor thickness reflects the depth of invasion more accurately than the interval between vessels.

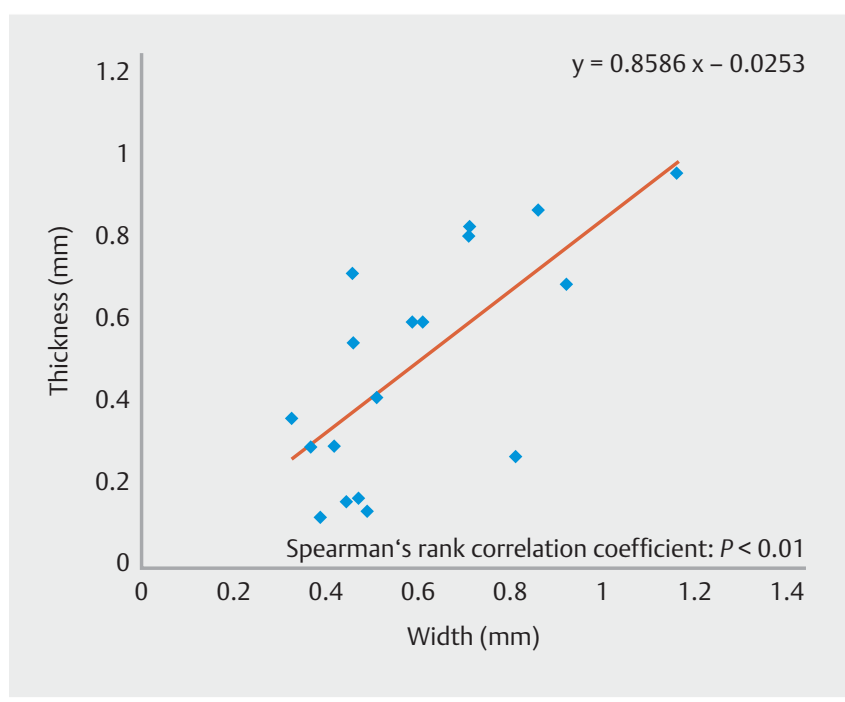

Fig. 5 Relationship between the width and thickness of the avascular areas.

Thus, it is reasonable to define AVA as an avascular or hypovascular area in the Japan Esophageal Society's classification of magnifying endoscopy findings. The concept of this definition indicates that the interval between vessels, or AVA width, cannot reflect the tumor depth when the tumor becomes large, invades a deeper layer, and develops neovascularization. However, determining the range of the hypovascular area is difficult. Thus, there is a possibility that the AVA thickness will help to assess the depth of invasion of the tumor, although it is impossible to accurately measure the thickness of the AVA in vivo.

It has long been known before magnifying endoscopy became popular that the thickness of superficial esophageal squamous cell carcinomas reflects invasion depth. Ohashi et al. [15] have reported that the tumor thickness and diameter of invasion were correlated with submucosal invasion. They concluded that a classification based on gross type, thickness, depth of depressed lesions, shape of elevated lesions, and invasion patterns should be evaluated to differentiate between M3 
and SM tumors. In the case of AVAs, an expansive growth type, our results support these findings in terms of the microvascular structure and invasion type.

\section{Conclusion}

Magnifying endoscopy can be used to image the microvascular structure of the concerned area and to assess the histopathological features accurately. Further investigations are needed to elucidate the changes in AVA vascular patterns occurring during tumor progression from M2 to M3 or SM cancers.

\section{Acknowledgements}

The authors would like to thank Editage (www.editage.jp) for English language editing.

\section{Competing interests}

None

\section{References}

[1] Inoue H, Honda T, Yoshida T et al. Ultra-high magnification endoscopy of the normal esophageal mucosa. Dig Endosc 1996; 8: 134 138

[2] Inoue $\mathrm{H}$, Honda T, Nagai K et al. Ultra-high magnification endoscopic observation of carcinoma in situ. Dig Endosc 1997; 1: 16-18

[3] Kumagai Y, Inoue H, Nagai K et al. Magnifying endoscopy, stereoscopic microscopy and the microvascular architecture of the superficial esophageal carcinoma. Endoscopy 2002; 34: 369-375
[4] Arima M, Arima H, Tada M et al. Diagnostic accuracy of tumor staging and treatment outcomes in patients with superficial esophageal cancer. Esophagus 2007; 4: 145-153

[5] Ishihara R, Inoue $T$, Uedo $N$ et al. Significance of each narrow-band image finding in diagnosing squamous mucosal high grade neoplasia of the esophagus. J Gastroenterol Hepatol 2010; 25: 1410-1415

[6] Kumagai Y, Toi M, Inoue H. Dynamism of tumour vasculature in the early phase of cancer progression: outcomes from oesophageal cancer research. Lancet Oncol 2002; 10: 604-610

[7] Arima M, Tada M, Arima H. Evaluation of microvascular patterns of superficial esophageal cancers by magnifying endoscopy. Esophagus 2005; 2: $191-197$

[8] Ebi M, Shimura T, Murakami K et al. Comparison of staging diagnosis by two magnifying endoscopy classification for superficial oesophageal cancer. Dig Liver Dis 2012; 44: 940 - 944

[9] Oyama T. Magnified endoscopic classification for superficial esophageal squamous cell carcinoma: classification of the Japan Esophageal Society. Stomach Intestine 2014; 49: 148-152

[10] Inoue M, Miyake Y, Odaka T et al. Objective evaluation of visibility in virtual chromoendoscopy for esophageal squamous carcinoma using a color difference formula. J Biomed Opt 2010; 15: 056019

[11] Osawa H, Yamamoto H, Miura Y et al. Blue laser imaging provides excellent endoscopic images of upper gastrointestinal lesions. Video J Encyclopedia GI Endosc 2014; 1: 607-610

[12] Kaneko K, Oono Y, Yano T et al. Effect of novel bright image enhanced endoscopy using blue laser imaging (BLI). Endosc Int Open 2014; 02: E212-E219

[13] Ishihara R, Aoi K, Matsuura N. Significance of AVA for prediction of infiltration depth of esophageal cancer. Stomach Intestine 2014; 49: $204-211$

[14] Folkman J. Clinical applications of research on angiogenesis. N Engl ] Med 1995; 333: $1757-1763$

[15] Ohashi K, Momma K, Yamada Y et al. Vertical and horizontal growth features of superficial esophageal squamous cell carcinomas: histopathological evaluation of endoscopically resected specimens. Virchows Arch 2002; 441: 350 - 357 